\title{
Isoscalar Hamiltonians for light atomic nuclei
}

\author{
G. P. Kamuntavičius ${ }^{a, b, c}$, P. Navrátil ${ }^{a, d}$, B. R. Barrett ${ }^{a}$, \\ G. Sapragonaite ${ }^{b}$, and R. K. Kalinauskas ${ }^{c}$ \\ ${ }^{a}$ Department of Physics, University of Arizona, Tucson, Arizona 85721 \\ ${ }^{b}$ Vytautas Magnus University, Kaunas LT-3000, Lithuania \\ ${ }^{c}$ Institute of Physics, Vilnius LT-2600, Lithuania \\ ${ }^{d}$ Institute of Nuclear Physics, Academy of Sciences of the Czech \\ Republic, \\ 25068 Rež near Prague, Czech Republic
}

(October 19, 2018)

\begin{abstract}
The charge-dependent realistic nuclear Hamiltonian for a nucleus, composed of neutrons and protons, can be successfully approximated by a chargeindependent one. The parameters of such a Hamiltonian, i.e., the nucleon mass and the NN potential, depend upon the mass number A, charge $\mathrm{Z}$ and isospin quantum number $\mathrm{T}$ of state of the studied nucleus.
\end{abstract}

Typeset using REVTEX 


\section{INTRODUCTION}

The model of the atomic nucleus as a system composed of neutrons and protons has been studied for over sixty years and has been found to provide a more-or-less adequate description. By considering the constituents of the nucleus as structureless identical fermions (i.e., nucleons), we obtain a very useful formalism, which leads to significant simplifications of our understanding and description of nuclear structure. These simplifications follow from the fact that the neutron and the proton have similar masses and that the potentials of the strong neutron-neutron (nn), neutron-proton (np) and proton-proton (pp) interactions are also very similar. Over the intervening years, simple models without correlations, such as the nuclear shell model, have been unable to distinguish between different parametrizations of the nucleon-nucleon (NN) interaction or to detect details in the definition of the mass of the nucleon.

During the last decade, however, theoretical techniques for calculating few-nucleon systems and the lightest atomic nuclei have advanced to the point that the quality of the realistic NN potential is starting to play an important role [1]. This necessarily involves the introduction of charge dependence into the NN interaction. The most-recent realistic potentials, such as the Nijmegen [2], Argonne [3] or CD-Bonn [4], are charge-dependent. On the one hand, the charge dependence of the nuclear Hamiltonian complicates the description, but on the other, the deviations of charge-dependent potentials from the charge-independent ones, although non-negligible, are not very large. As a consequence, modern calculations of light nuclei use as a starting point a Hamiltonian preserving the isospin quantum number and include charge-dependent effects perturbatively later on. The charge dependence of the kinetic and potential energies of a real nucleus, however, can only be successfully taken into account perturbatively, when the zeroth-order, charge-independent, isoscalar nuclear Hamiltonian is as close as possible to the charge-dependent one. We present here a method of construction for such a Hamiltonian.

In Sections II and III we show that the kinetic-energy operator of an atomic nucleus composed of neutrons and protons can be precisely approximated by a corresponding operator for a nucleus composed of nucleons, when the nucleon mass is defined as the mean value of the quantum-mechanical operator for the nucleon mass. The nucleon mass obtained in this way depends on the mass number A and charge $\mathrm{Z}$ of the studied nucleus.

In Section IV we investigate the problem concerning the definition of the NN interaction. For the sake of simplicity, we consider potentials, defined separately in different two-nucleon channels, such as, the Reid'68 [5] and Nijmegen [2] potentials. The generalization of results given by other realistic charge-dependent potentials, such as the CD-Bonn [4] and Argonne18 [3] potentials, is straightforward. It is shown that the best NN potential is a linear combination of the nn, np, and pp potentials with coefficients, dependent not only on the nucleus studied, i.e., $A$ and $Z$, but also on the isospin quantum number $T$ of the state under consideration. So, the isoscalar Hamiltonians that we introduce are different for different nuclei and even for different states of the same nucleus. The derived values for the nucleon mass and the NN interaction must satisfy minimal requirements (e.g., for systems composed only of neutrons, the nucleon mass formula must give the neutron mass and the NN interaction must reduce to the potential of the neutron-neutron interaction) and must also be equal to the corresponding values for non-trivial cases suggested for three-nucleon 
nuclei in earlier publications [6], [7].

In Section $\mathrm{V}$ we present numerical tests of our recommendations and give our conclusions in Section VI.

\section{THE MASS OF THE NUCLEON}

The nonrelativistic kinetic energy operator of the atomic nucleus equals

$$
K=-\frac{\hbar^{2}}{2 m_{p}} \sum_{i=1}^{Z} \nabla_{i}^{2}-\frac{\hbar^{2}}{2 m_{n}} \sum_{i=Z+1}^{A} \nabla_{i}^{2}+\frac{\hbar^{2}}{2 M} \nabla_{\mathbf{R}}^{2}
$$

where $m_{p}$ and $m_{n}$ are the proton and the neutron masses, respectively; $M=Z m_{p}+N m_{n}$ is the mass of the nucleus and

$$
\mathbf{R}=\frac{1}{M}\left(\sum_{i=1}^{Z} m_{p} \mathbf{r}_{i}+\sum_{i=Z+1}^{A} m_{n} \mathbf{r}_{i}\right)
$$

is the radius-vector of the center-of-mass of the nucleus .

The kinetic energy operator of the nucleus, as a system of nucleons is simpler:

$$
K^{0}=-\frac{\hbar^{2}}{2 m} \sum_{i=1}^{A} \nabla_{i}^{2}+\frac{\hbar^{2}}{2 A m} \nabla_{\mathbf{R}_{0}}^{2}
$$

where $m$ is the nucleon mass and

$$
\mathbf{R}_{0}=\frac{1}{A} \sum_{i=1}^{A} \mathbf{r}_{i}
$$

is the center-of-mass radius-vector of the nucleus composed of nucleons.

Obviously, the operators $K$ and $K^{0}$ are different. In particular, the expression for $K^{0}$ contains the parameter $m$, which, in many applications, is defined as an average of the proton and neutron masses; i.e.,

$$
\bar{m}=\frac{m_{n}+m_{p}}{2}
$$

On the other hand, calculations for a neutron and proton system involve a transformation to relative and center-of-mass coordinates and a mass equal to twice the reduced mass

$$
\mu=\frac{2 m_{n} m_{p}}{m_{n}+m_{p}}
$$

While in the three nucleon case, it is sometimes assumed that ${ }^{3} \mathrm{H}$ and ${ }^{3} \mathrm{He}$ are composed of identical particles, each of mass $\frac{1}{3}\left(2 m_{n}+m_{p}\right), \frac{1}{3}\left(m_{n}+2 m_{p}\right)$ [6], respectively.

At first glance, it looks as though we can define the optimal kinetic energy operator, which is as close as possible to a charge-dependent one, yet charge-independent, by properly 
defining the mass of the nucleon in $K^{0}$. However, the nucleon mass is not really a free parameter. It has to be defined as the mean value of a nucleon mass operator:

$$
\hat{m}=\frac{1}{A} \sum_{i=1}^{A} m(i)=\frac{1}{A} \sum_{i=1}^{A}\left(m_{n} P_{n}(i)+m_{p} P_{p}(i)\right),
$$

where $m(i)$ is the mass of an $i$-th nucleon and

$$
P_{p}(i)=\frac{1}{2}-\tau_{z}(i), \quad P_{n}(i)=\frac{1}{2}+\tau_{z}(i)
$$

are projectors upon proton and neutron states.

The mass of the nucleon in the nucleus $(A, Z)$ can be defined exactly, because in the $A$-nucleon state, with a defined isospin projection, $M_{T}=\frac{1}{2}(N-Z)$ :

$$
\begin{aligned}
m & =\left\langle M_{T}|\hat{m}| M_{T}\right\rangle=\left\langle M_{T}\left|\frac{1}{A} \sum_{i=1}^{A} m(i)\right| M_{T}\right\rangle \\
& =\left\langle M_{T}\left|\frac{m_{n}+m_{p}}{2}+\frac{\left(m_{n}-m_{p}\right)}{A} \sum_{i=1}^{A} \tau_{z}(i)\right| M_{T}\right\rangle \\
& =\frac{m_{n}+m_{p}}{2}+\frac{\left(m_{n}-m_{p}\right)}{A}\left\langle M_{T}\left|T_{z}\right| M_{T}\right\rangle \\
& =\frac{m_{n}+m_{p}}{2}+\frac{\left(m_{n}-m_{p}\right)}{A} M_{T},
\end{aligned}
$$

or, in other words,

$$
m=\frac{1}{A}\left(N m_{n}+Z m_{p}\right)
$$

This definition of the nucleon mass coincides with that used in the above-mentioned threenucleon case. For light nuclei with $N=Z$, it equals the mean value of the neutron and proton masses. For two nucleon systems it coincides with twice the reduced mass in cases of two protons or two neutrons and is very close to twice the neutron-proton reduced mass because

$$
\mu-\bar{m}=\frac{2 m_{n} m_{p}}{m_{n}+m_{p}}-\frac{m_{n}+m_{p}}{2}=-\bar{m} \delta^{2} \approx-4.5 \times 10^{-4} \frac{M e V}{c^{2}},
$$

where the widely used small parameter is:

$$
\delta=\frac{m_{n}-m_{p}}{m_{n}+m_{p}} \approx 6.887 \times 10^{-4} .
$$

Obviously, the difference between $\mu$ and $\bar{m}$ is negligible. Realistic NN potentials, defined by using a reduced mass for the two nucleons, cannot change significantly when the nucleon mass $m$, defined for the two-nucleon system, is used instead of $\mu$. 


\section{INTRINSIC VARIABLES AND OPERATORS}

A direct comparison of the operators $K^{0}$ and $K$ is impossible due to different neutron and proton masses and different definitions of center-of-mass operators. On the one hand, both operators, $K^{0}$ and $K$, have redundant variables, because they are functions not only of one nucleon but also of center-of-mass variables. On the other hand, both operators are intrinsic; this means dependent only on intrinsic, translationally invariant variables and independent of center-of-mass radius vectors. In order to compare them, it is necessary to introduce intrinsic variables.

The Jacobi variables $\eta_{0}, \eta_{1}, \ldots \eta_{A-1}$ for particles with equal masses are well-known (see [8], [9] and references therein). They can be defined by a corresponding Jacobi tree as an orthonormal set. The expressions for $\eta_{\alpha}$ in terms of $\mathbf{r}_{i}$ and vice versa are as follows:

$$
\eta_{\alpha}=\sum_{i=1}^{A} a_{\alpha, i} \mathbf{r}_{i}, \quad \mathbf{r}_{i}=\sum_{\alpha=0}^{A-1} \eta_{\alpha} a_{\alpha, i} .
$$

Here $\mathbf{a}$ is an orthogonal $(A \times A)$ matrix :

$$
\sum_{i=1}^{A} a_{\alpha, i} a_{\beta, i}=\delta_{\alpha, \beta}, \quad \sum_{\alpha=0}^{A-1} a_{\alpha, i} a_{\alpha, j}=\delta_{i, j} .
$$

The translational invariance of the intrinsic variables $\eta_{1}, \ldots \eta_{A-1}$ along with orthogonality gives the following conditions for the matrix $\mathbf{a}$ :

$$
\sum_{i=1}^{A} a_{\alpha, i}=\sqrt{A} \delta_{\alpha, 0}, \quad a_{0, i}=\frac{1}{\sqrt{A}} .
$$

Using these variables, we can rewrite the operator (2) as follows:

$$
K^{0}=-\frac{\hbar^{2}}{2 m} \sum_{\alpha=1}^{A-1} \nabla_{\eta_{\alpha}}^{2}
$$

The analogous presentation for $K$ requires Jacobi variables for particles with different proton and neutron masses. Let us introduce Jacobi variables in the general case, in which the masses of all particles are different. The reduction to protons and neutrons will then be straightforward. These variables can be defined by the same kind of Jacobi tree, as in the previous case.

The Jacobi tree by definition has $(2 A-1)$ vertices, $A$ of which are vertices of the first degree (the degree of a vertex is defined as the number of edges matching in this point). They should be arranged in a line and marked with the one-particle radius-vectors $\mathbf{r}_{1}, \mathbf{r}_{2}, \ldots, \mathbf{r}_{A}$ . In case of particles with different masses, however, the vertices of the first degree of the tree have to be marked not only by one-particle radius-vectors but also by corresponding masses $m_{1}, m_{2}, \ldots, m_{A}$. The remaining $(A-1)$ vertices (situated below the first group) determine the Jacobi coordinates $\xi_{\alpha}(\alpha=1,2, \ldots, A-1)$.The degree of each equals three, except for the deepest one which equals two. Contrary to the case of equivalent particles, the 
orthogonal transformation can now be defined only between Jacobi variables $\xi_{0}, \xi_{1}, \ldots, \xi_{A-1}$ and modified one-particle variables $\mathbf{x}_{1}, \mathbf{x}_{2}, \ldots, \mathbf{x}_{A}$, where $\mathbf{x}_{i}=\sqrt{m_{i}} \mathbf{r}_{i}$. For the $\alpha$-th vertex the Jacobi coordinate is

$$
\xi_{\alpha}=\sqrt{\frac{m_{L}^{(\alpha)} m_{R}^{(\alpha)}}{m_{L}^{(\alpha)}+m_{R}^{(\alpha)}}}\left[\frac{1}{m_{L}^{(\alpha)}} \sum_{i \in\{L\}} \sqrt{m_{i}} \mathbf{x}_{i}-\frac{1}{m_{R}^{(\alpha)}} \sum_{i \in\{R\}} \sqrt{m_{i}} \mathbf{x}_{i}\right], \quad(\alpha=1,2, \ldots, A-1) .
$$

Here $\{L\}$ is a manifold of the first-degree vertices, which could be reached while moving from the $\alpha$-th vertex upwards along the left edge, while $\{R\}$ denotes the same for the right edge. Obviously, the new Jacobi variables, $\xi_{\alpha}(\alpha=1,2, \ldots, A-1)$, like the corresponding old ones, $\eta_{\alpha}(\alpha=1,2, \ldots, A-1)$, are translationally invariant, because they are defined as properly normalized radius vectors between the centers-of-mass of two subtrees, defined by left and right edges of the tree, as they are seen from the $\alpha$-th vertex. The masses of left and right Jacobi clusters are defined in the following way:

$$
m_{L}^{(\alpha)}=\sum_{i \in\{L\}} m_{i}, \quad m_{R}^{(\alpha)}=\sum_{i \in\{R\}} m_{i} .
$$

This set has to be completed by a zero Jacobi variable, situated on the left side of a seconddegree vertex and proportional to a center-of-mass radius vector:

$$
\xi_{0}=\frac{1}{\sqrt{M}} \sum_{i=1}^{A} \sqrt{m_{i}} \mathbf{x}_{i}
$$

where $M=\sum_{i=1}^{A} m_{i}$ is the mass of the system. In general,

$$
\xi_{\alpha}=\sum_{i=1}^{A} b_{\alpha, i}\left(m_{1}, m_{2, \ldots}, m_{A}\right) \mathbf{x}_{i}, \quad(\alpha=0,1, \ldots, A-1),
$$

where $\mathbf{b}$ is an orthogonal $(A \times A)$ matrix. In the case of equivalent particles, this transformation coincides with that defined in Eq. (8) multiplied by $\sqrt{m}$. The proof of the orthogonality of these transformations is given in Appendix A.

The tree sample for five particles is presented in Fig. 1. The matrix $\mathbf{b}$ for this tree equals

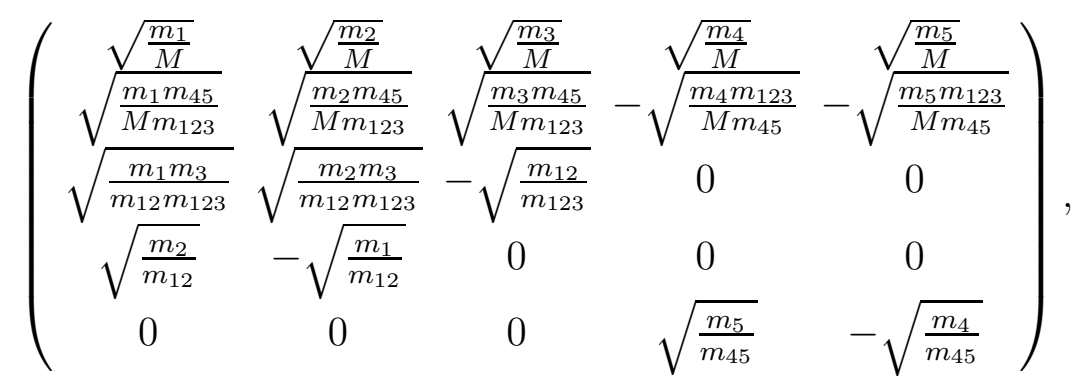

where $m_{j \ldots k}=\sum_{i=j}^{k} m_{i} ; M=m_{12345}$. 
Due to the orthogonality of the transformation from one-particle variables $\mathbf{x}_{1}, \mathbf{x}_{2}, \ldots, \mathbf{x}_{A}$ to a system of Jacobi variables $\xi_{0}, \xi_{1}, \ldots, \xi_{A-1}$, the transformation from one set to another set of Jacobi variables, defined by different trees, could be determined in the same way.

The kinetic energy operator (1) in terms of these new variables can be rewritten as

$$
K=-\frac{\hbar^{2}}{2} \sum_{\alpha=1}^{A-1} \nabla_{\xi_{\alpha}}^{2} .
$$

A comparison of operators Eq.(11) and Eq.(15) requires the transformation matrix between the sets $\xi_{0}, \xi_{1}, \ldots, \xi_{A-1}$ and $\eta_{0}, \eta_{1}, \ldots \eta_{A-1}$. The result cannot depend on the choice of the Jacobi tree, so we can choose the simplest one, given in Fig. 2, where protons are marked by the numbers $1, \ldots, Z$ and neutrons by the numbers $Z+1, \ldots, Z+N=A$. In such a case



$$
\begin{aligned}
& =\sum_{\beta=0}^{A-1}\left(\sum_{i=1}^{A} b_{\alpha, i}\left(m_{1}, m_{2, \cdots}, m_{A}\right) \sqrt{m_{i}} a_{\beta, i}\right) \eta_{\beta} .
\end{aligned}
$$

Due to the multipliers $\sqrt{m_{i}}$, the matrix of the last transformation is not an orthogonal matrix. This gives the following expression for $K$ in terms of variables $\eta_{\alpha}$ :

$$
\begin{aligned}
K & =-\frac{\hbar^{2}}{2}\left[\frac{Z N\left(m_{n}-m_{p}\right)^{2}}{A M m_{n} m_{p}} \nabla_{\eta_{0}}^{2}+\frac{2 \sqrt{Z N}\left(m_{n}-m_{p}\right)}{A m_{n} m_{p}}\left(\nabla_{\eta_{0}} \cdot \nabla_{\eta_{1}}\right)\right. \\
& \left.+\frac{M}{A m_{n} m_{p}} \nabla_{\eta_{1}}^{2}+\frac{1}{m_{p}} \sum_{\alpha=2}^{Z} \nabla_{\eta_{\alpha}}^{2}+\frac{1}{m_{n}} \sum_{\alpha=Z+1}^{A-1} \nabla_{\eta_{\alpha}}^{2}\right] .
\end{aligned}
$$

So, the neutron and proton mass difference causes the intrinsic kinetic energy operator to depend upon the center-of-mass variable. The center-of-mass starts to move in an atomic nucleus composed of nucleons instead of real protons and neutrons. However, this effect is not significant, because the first term, proportional to $\nabla_{\eta_{0}}^{2}$, has a very small coefficient, while the expectation value of the second term, proportional to $\left(\nabla_{\eta_{0}} \cdot \nabla_{\eta_{1}}\right)$, vanishes either when the excitations of the center-of-mass of a nucleus are under control or when the wave function is intrinsic.

The difference in the kinetic-energy operators for neutron-proton and nucleon nuclei is, thus, given by

$$
\begin{gathered}
K-K^{0}=-\frac{\hbar^{2}}{2 m}\left[\delta^{2} \frac{Z N}{A} \frac{\left(m_{n}+m_{p}\right)^{2}}{M m_{n} m_{p}} \nabla_{\eta_{0}}^{2}+2 \delta \frac{\sqrt{Z N}}{A} \frac{m_{n}+m_{p}}{m_{n} m_{p}}\left(\nabla_{\eta_{0}} \cdot \nabla_{\eta_{1}}\right)\right. \\
\left.+\left(\frac{M m}{A m_{n} m_{p}}-1\right) \nabla_{\eta_{1}}^{2}+\left(\frac{m}{m_{p}}-1\right) \sum_{\alpha=2}^{Z} \nabla_{\eta_{\alpha}}^{2}+\left(\frac{m}{m_{n}}-1\right) \sum_{\alpha=Z+1}^{A-1} \nabla_{\eta_{\alpha}}^{2}\right],
\end{gathered}
$$

where $\delta$ is the parameter (7).

From the above expression, it is obvious that the mean value of the difference in kinetic energies, estimated using intrinsic wave functions, equals the sum of the mean values of the last three terms and can be rewritten as:

$$
\begin{aligned}
\left\langle\mathcal{E} J \Pi M_{T}\left|K-K^{0}\right| \mathcal{E} J \Pi M_{T}\right\rangle= & {\left[\left(\frac{M m}{A m_{n} m_{p}}-1\right)+\left(\frac{m}{m_{p}}-1\right)(Z-1)+\left(\frac{m}{m_{n}}-1\right)(N-1)\right] } \\
& \times\left\langle\mathcal{E} J \Pi M_{T}\left|-\frac{\hbar^{2}}{2 m} \nabla_{\eta_{\alpha}}^{2}\right| \mathcal{E} J \Pi M_{T}\right\rangle,
\end{aligned}
$$


because the mean value of $\nabla_{\eta_{\alpha}}^{2}$ is independent of $\alpha$ (see Appendix B). The expression given in square brackets equals

$$
\frac{Z N(A-1)}{A^{2}} \frac{\left(m_{n}-m_{p}\right)^{2}}{m_{n} m_{p}} \approx \frac{4 Z N(A-1)}{A^{2}} \delta^{2}
$$

while the matrix element has the same order as the kinetic energy of a single nucleon in a light nucleus ( $\leq 40 \mathrm{MeV}$, see [10]). Consequently,

$$
\left\langle K-K^{0}\right\rangle \lesssim 10^{-5} \mathrm{MeV}
$$

This means that by using the defined value of the nucleon mass (6), we can account for the $n-p$ mass difference in the expression for the intrinsic kinetic energy without any problems. In cases, when the mean value of the neutron and proton masses (3) is used instead of the defined value of the nucleon mass, this difference of expectation values (16) is proportional to $\delta$ and can cause a noticeable difference in the kinetic energies $K$ and $K^{0}$.

\section{THE POTENTIAL OF THE NUCLEON - NUCLEON INTERACTION}

The Hamiltonian of an atomic nucleus with a charge independence and charge symmetry breaking NN potential has the form

$$
H=K+V
$$

where $K$ is the translationally invariant operator of kinetic energy, Eq. (11), and

$$
V=\sum_{i, k=1(i<k)}^{A}\left[V_{p p}(i, k) P_{p}(i) P_{p}(k)+V_{n p}(i, k) P_{n}(i) P_{p}(k)+V_{n n}(i, k) P_{n}(i) P_{n}(k)\right]
$$

is the potential-energy operator (the Coulomb interaction is included in $V_{p p}(i, k)$ ).

It is useful to employ the isospin formalism and the partial-waves expansion in order to simplify the definition of the NN potential, i.e.,

$$
\begin{aligned}
V(i, k) & \equiv V\left(\mathbf{r}_{i k} \sigma_{i} \tau_{i} \sigma_{k} \tau_{k}\right) \\
& =\sum_{j \pi t m_{t}} V^{j \pi t m_{t}}\left(r_{i k}\right) P_{j \pi t m_{t}}\left(\theta_{i k} \phi_{i k} \sigma_{i} \tau_{i} \sigma_{k} \tau_{k}\right),
\end{aligned}
$$

where $\mathbf{r}_{i k} \equiv\left(r_{i k} \theta_{i k} \phi_{i k}\right)=\mathbf{r}_{i}-\mathbf{r}_{k}$ is the difference of radius-vectors of the $\mathrm{i}$-th and the $\mathrm{k}$-th nucleons; $\sigma_{i} \tau_{i}$ are the spin and isospin variables of the $i$-th nucleon, respectively; $j \pi t$ are the quantum numbers - total momentum, parity and isospin - of the two nucleon state; and $P_{j \pi t m_{t}}\left(\theta_{i k} \phi_{i k} \sigma_{i} \tau_{i} \sigma_{k} \tau_{k}\right)$ is the projection operator on this state. The correspondence between our notation, $j \pi t$, and conventional spectroscopic identifiers ${ }^{2 s+1} L_{j}$ is:

$$
0^{+} 1 \sim^{1} S_{0}, \quad 0^{-} 1 \sim^{3} P_{0,} \quad 1^{+} 0 \sim^{3} S_{1}-^{3} D_{1}, \quad 1^{-} 1 \sim^{3} P_{1}, \quad 1^{-} 0 \sim^{1} P_{1}, \ldots
$$

Also, $m_{t}$ stands for the two-nucleon isospin projection; $m_{t}=1$ corresponds to two neutrons; $m_{t}=0$, to neutron-proton; and $m_{t}=-1$, to two protons. Traditionally, NN potentials 
are constructed by fitting np $\left(m_{t}=0\right)$ data for $t=0$ states and either np $\left(m_{t}=0\right)$ or pp $\left(m_{t}=-1\right)$ data for $t=1$ states 10 .

In general, the realistic potentials can be different for $m_{t}=0, \pm 1$ in each twonucleon channel with $t=1$. To some extent it holds even for charge-symmetric potentials of the strong interaction, because the Coulomb interaction must to be included in $V^{j \pi(t=1)\left(m_{t}=-1\right)}\left(r_{i k}\right)$. In channels with $\pi=(-1)^{j}$ the potential $V^{j \pi t m_{t}}\left(r_{i k}\right)$ is one function of $r_{i k}$, when $t=0$, and three different functions of $r_{i k}$, when $t=1$ (the best example being $\left.{ }^{1} \mathrm{~S}_{0}\right)$. In channels with $\pi=(-1)^{j+1}$ and $t=0$, the potential $V^{j \pi t m_{t}}\left(r_{i k}\right)$ can be given as three different functions of $r_{i k}$, namely entries in a second-order symmetric matrix (such as the parametrization in the channel $1^{+} 0 \sim^{3} S_{1}-{ }^{3} D_{1}$, as given for the Reid68 [5] and Nijmegen [2] potentials) or nine different functions of $r_{i k}$, corresponding to different values of $m_{t}$ when $\pi=(-1)^{j+1}$ and $t=1$. The first channel of this kind is $2^{-} 1$. This parametrization, however, is performed as three different functions of $r_{i k}$. As mentioned earlier, they obviously are different for $m_{t}=-1$ in comparison to $m_{t}=0$ or $m_{t}=+1$, due to Coulomb interaction of protons.

The charge-independent Hamiltonian of an atomic nucleus composed of nucleons is

$$
H^{0}=K^{0}+V^{0}
$$

with the kinetic energy operator, defined in Eq.(2), and the charge-independent potentialenergy operator

$$
V^{0}=\sum_{i, k=1(i<k)}^{A} V^{0}(i, k)
$$

whose partial-wave expansion is given by

$$
\begin{aligned}
V^{0}(i, k) & \equiv V^{0}\left(\mathbf{r}_{i k} \sigma_{i} \tau_{i} \sigma_{k} \tau_{k}\right) \\
& =\sum_{j \pi t} V^{j \pi t}\left(r_{i k}\right) P_{j \pi t}\left(\theta_{i k} \phi_{i k} \sigma_{i} \tau_{i} \sigma_{k} \tau_{k}\right) .
\end{aligned}
$$

As was shown above, there are no problems with regard to the charge-dependence of the kinetic energy, because by taking the proper value of the nucleon mass, one can use $K^{0}$ instead of $K$ in the expression for $H$, due to the negligible difference between these two kinetic energy operators. In such a case, the Hamiltonian $H$ can be expressed in the form

$$
H=\sum_{i, k=1(i<k)}^{A} h(i, k),
$$

where

$$
h(i, k)=-\frac{\hbar^{2}}{2 m A}\left(\nabla_{i}-\nabla_{k}\right)^{2}+V\left(\mathbf{r}_{i}-\mathbf{r}_{k}, \sigma_{i} \tau_{i} \sigma_{k} \tau_{k}\right) .
$$

The exact expression for an arbitrary eigenvalue of such an operator is ( see [11], 12 and references therein) : 


$$
\mathcal{E}^{J \Pi M_{T}}=\langle H\rangle_{\mathcal{E}, \mathcal{E}}^{J \Pi M_{T}}=\frac{A(A-1)}{2} \sum_{\varepsilon, j \pi t m_{t}} \varepsilon^{j \pi t m_{t}} Q_{\varepsilon, j \pi t m_{t}}\left(\mathcal{E} J \Pi M_{T}\right),
$$

where the sum runs over all the two nucleon states $j \pi t$ and all eigenvalues $\varepsilon$ of the Reduced Hamiltonian (RH) operator

$$
h \equiv h(A-1, A)=-\frac{2 \hbar^{2}}{m A} \Delta_{\mathbf{r}}+V\left(\mathbf{r}, \sigma_{A-1} \tau_{A-1} \sigma_{A} \tau_{A}\right)
$$

with $\mathbf{r}=\mathbf{r}_{A-1}-\mathbf{r}_{A}$.

The $Q_{\varepsilon, j \pi t m_{t}}\left(\mathcal{E} J \Pi M_{T}\right)$ are diagonal entries of the intrinsic density matrix in terms of the $\mathrm{RH}$ eigenfunctions:

$$
\begin{aligned}
Q_{\varepsilon, j \pi t m_{t}}(\mathcal{E} J \Pi & \left.M_{T}\right) \equiv Q_{\varepsilon, j \pi t m_{t}, \varepsilon, j \pi t m_{t}}\left(\mathcal{E} J \Pi M_{T}\right) \\
Q_{\varepsilon, j \pi t m_{t}, \varepsilon^{\prime}, j^{\prime} \pi^{\prime} t^{\prime} m_{t}^{\prime}}\left(\mathcal{E} J \Pi M_{T}\right)= & \sum_{\sigma_{1} \tau_{1} \ldots \sigma_{A-1} \tau_{A-1} \sigma_{A} \tau_{A} \sigma_{A-1}^{\prime} \tau_{A-1}^{\prime} \sigma_{A}^{\prime} \tau_{A}^{\prime}} \int d \xi_{1} \ldots d \xi_{A-2} d \xi_{A-1} d \xi_{A-1}^{\prime} \\
& \times \psi_{\varepsilon, j \pi t m_{t}}^{+}\left(\xi_{A-1} \sigma_{A-1} \tau_{A-1} \sigma_{A} \tau_{A}\right) \\
& \times \Psi_{\mathcal{E} J \Pi M_{T}}\left(\xi_{1 \ldots} \ldots \xi_{A-2} \xi_{A-1} \sigma_{1} \tau_{1} \ldots \sigma_{A-1} \tau_{A-1} \sigma_{A} \tau_{A}\right) \\
& \times \Psi_{\mathcal{E} J \Pi M_{T}}^{+}\left(\xi_{1} \ldots \xi_{A-2} \xi_{A-1}^{\prime} \sigma_{1} \tau_{1} \ldots \sigma_{A-1}^{\prime} \tau_{A-1}^{\prime} \sigma_{A}^{\prime} \tau_{A}^{\prime}\right) \\
& \times \psi_{\varepsilon^{\prime}, j^{\prime} \pi^{\prime} t^{\prime} m_{t}^{\prime}}\left(\xi_{A-1}^{\prime} \sigma_{A-1}^{\prime} \tau_{A-1}^{\prime} \sigma_{A}^{\prime} \tau_{A}^{\prime}\right)
\end{aligned}
$$

where $\Psi_{\mathcal{E} J \Pi M_{T}}\left(\xi_{1} \ldots \xi_{A-1} \sigma_{1} \tau_{1} \ldots \sigma_{A} \tau_{A}\right)$ is the intrinsic wave-function of the nucleus.

According to the definition of the density matrix

$$
Q_{\varepsilon, j \pi t m_{t}}\left(\mathcal{E} J \Pi M_{T}\right) \geq 0, \quad \text { and } \sum_{\varepsilon, j \pi t m_{t}} Q_{\varepsilon, j \pi t m_{t}}\left(\mathcal{E} J \Pi M_{T}\right)=1 .
$$

These are probabilities of definite states of two nucleon relative motion, $\quad \varepsilon j \pi t m_{t}$, in the state under investigation, $\mathcal{E} J \Pi M_{T}$, i.e., the state specified by the exact quantum numbers - energy, total momentum, parity and isospin projection of the nucleus $(A, Z)$.

The expression for the eigenvalue of the Hamiltonian

$$
H^{0}=\sum_{i, k=1(i<k)}^{A} h^{0}(i, k)
$$

where

$$
h^{0}(i, k)=-\frac{\hbar^{2}}{2 m A}\left(\nabla_{i}-\nabla_{k}\right)^{2}+V^{0}\left(\mathbf{r}_{i}-\mathbf{r}_{k}, \sigma_{i} \tau_{i} \sigma_{k} \tau_{k}\right)
$$

is very similar to that given by Eq. (23):

$$
\mathcal{E}^{J \Pi T}=\left\langle H^{0}\right\rangle_{\mathcal{E}, \mathcal{E}}^{J \Pi T}=\frac{A(A-1)}{2} \sum_{\varepsilon, j \pi t} \varepsilon^{j \pi t} Q_{\varepsilon, j \pi t}(\mathcal{E} J \Pi T)
$$


but is slightly simpler, because the corresponding NN potential is charge-independent. As a consequence, the total isospin $T$ is an exact quantum number. Moreover, in such a case, the eigenvalues are the same for all nuclei of the isospin multiplet and, hence, independent on $M_{T}$. Here

$$
h^{0} \equiv h^{0}(A-1, A)=-\frac{2 \hbar^{2}}{m A} \Delta_{\mathbf{r}}+V^{0}\left(\mathbf{r}, \sigma_{A-1} \tau_{A-1} \sigma_{A} \tau_{A}\right)
$$

A straightforward comparison of the eigenvalues, Eqs. (23) and (30), is impossible, as eigenfunctions of the Hamiltonians, $H$ and $H^{0}$, do not coincide. Eigenvalues and eigenfunctions of the corresponding Reduced Hamiltonians (Eqs. (24) and (31)) are also different. To compare them, it is necessary to define kind of equivalent potential $V^{0}$, for which we are looking. From partial-wave decompositions of the potentials, Eqs. (17) and (20), it follows, that the realistic potential in the states with $t=0$ is the same in both cases, because $m_{t}$ can take only the value zero. If $t=1$, there are three different pairs of nucleons $\left(m_{t}=0, \pm 1\right)$ and the NN potential $V^{j \pi t m_{t}}\left(r_{i k}\right)$ is different in each case. Therefore, the symmetric, charge-independent potential in an arbitrary two-nucleon state can be defined as the sum of these charge-dependent potentials:

$$
V^{j \pi t}\left(r_{i k}\right)=\sum_{m_{t}} c_{t m_{t}} V^{j \pi t m_{t}}\left(r_{i k}\right)
$$

where the $c_{t m_{t}}$ are normalized coefficients:

$$
\sum_{m_{t}} c_{t m_{t}}=1
$$

The optimal values of these coefficients are (see Appendix C):

$$
\begin{aligned}
c_{00} & =1 \\
c_{1-1} & =\frac{\left(A-2 M_{T}\right)\left(A-2 M_{T}-2\right)}{3 A(A-2)+4 T(T+1)}=\frac{4 Z(Z-1)}{3 A(A-2)+4 T(T+1)}, \\
c_{10} & =\frac{A(A-2)+4 T(T+1)-8 M_{T}^{2}}{3 A(A-2)+4 T(T+1)}=\frac{8 N Z-A(A+2)+4 T(T+1)}{3 A(A-2)+4 T(T+1)}, \\
c_{1+1} & =\frac{\left(A+2 M_{T}\right)\left(A+2 M_{T}-2\right)}{3 A(A-2)+4 T(T+1)}=\frac{4 N(N-1)}{3 A(A-2)+4 T(T+1)} .
\end{aligned}
$$

These values, obtained as a result of consideration of probability distributions, have a rather simple interpretation. Having in mind the definition of marginal probabilities, as given in [11], and the definition of the $c_{t m_{t}}$, as given in (C5), we find that these coefficients equal the number of pairs of nucleons with the isospin and corresponding projection quantum numbers, $t$ and $m_{t}$, divided by the number of pairs with the isospin quantum number $t$.

Finally, the charge independent Hamiltonian can be expressed in the form

$$
H^{0}\left(A, T, M_{T}\right)=\sum_{i, k=1(i<k)}^{A} h^{0}\left(A, T, M_{T} ; i, k\right),
$$

where 


$$
h^{0}\left(A, T, M_{T} ; i, k\right)=-\frac{\hbar^{2}}{2 m\left(A, M_{T}\right) A}\left(\nabla_{i}-\nabla_{k}\right)^{2}+V^{0}\left(A, T, M_{T} ; \mathbf{r}_{i}-\mathbf{r}_{k}, \sigma_{i} \tau_{i} \sigma_{k} \tau_{k}\right) .
$$

The eigenvalue of $H^{0}\left(A, T, M_{T}\right)$ is very similar to that given by Eq. (30), but now has the form

$$
\mathcal{E}^{J \Pi T}\left(A, T, M_{T}\right)=\left\langle H^{0}\left(A, T, M_{T}\right)\right\rangle_{\mathcal{E}, \mathcal{E}}^{J \Pi T}=\frac{A(A-1)}{2} \sum_{\varepsilon, j \pi t} \varepsilon^{j \pi t}\left(A, T, M_{T}\right) Q_{\varepsilon, j \pi t}(\mathcal{E} J \Pi T) .
$$

From the last expression we observe that our result for a charge-independent Hamiltonian, which includes charge dependent effects (e.g., Coulomb interaction, charge dependence of the potential and the neutron and proton mass difference), is a function of the parameters $A, T$, and $M_{T}$. Comparing with the exact expression (23), we observe that isospin now appears as an exact quantum number and the density matrix is independent of $M_{T}$. Consequently, the density matrix is the same for all members of an isospin multiplet, as it must be for a charge independent Hamiltonian. Contrary to the commonly used expression for charge-independent potentials, i.e., Eq. (30), the present effective Hamiltonians (Eq. (38)) are different for different nuclei of an isospin multiplet and for states of the same nucleus with different values of total isospin $T$.

\section{NUMERICAL TESTS}

The effective Hamiltonians (Eq. (38)), obtained above, agree with the well-known, trivial isoscalar Hamiltonians for nuclear matter and for states of finite nuclei with total isospin $T=0$, because, in this case, the nucleon mass equals half of neutron and proton masses and the effective potential is the average of the three potentials. However, even in this case, the recommendations given above allow us to express in isoscalar form the Coulomb interaction of the protons. In the light-nuclei region for isospin multiplets with $T \neq 0$, the values of the nucleon mass and the effective potential are different for nuclei with different $M_{T}$ values.

For the triton $\left(A=3, T=1 / 2, M_{T}=1 / 2\right)$, for example, the mass of the nucleon is

$$
m=\frac{2}{3} m_{n}+\frac{1}{3} m_{p}
$$

and the equivalent charge-independent potential is

$$
V^{j \pi(t=1)}(r)=\frac{1}{3} V^{j \pi(t=1)\left(m_{t}=0\right)}(r)+\frac{2}{3} V^{j \pi(t=1)\left(m_{t}=1\right)}(r),
$$

or simply

$$
V(r)=\frac{1}{3} V_{n p}^{t=1}(r)+\frac{2}{3} V_{n n}(r)
$$

which agrees with the results obtained in [6] using the same nucleon mass and in [7] for the charge-independent potential in the ${ }^{1} S_{0}$ state. 
For ${ }^{3} \mathrm{He}\left(A=3, T=1 / 2, M_{T}=-1 / 2\right)$ the mass of nucleon equals

$$
m=\frac{1}{3} m_{n}+\frac{2}{3} m_{p}
$$

The equivalent charge-independent potential is

$$
V^{j \pi(t=1)}(r)=\frac{1}{3} V^{j \pi(t=1)\left(m_{t}=0\right)}(r)+\frac{2}{3} V^{j \pi(t=1)\left(m_{t}=-1\right)}(r),
$$

with the Coulomb interaction included in the last term.

In order to test the results obtained in section IV, we performed several calculations in the framework of the large-basis, no-core shell-model approach [15], using interactions that directly break isospin as well as the corresponding NN interactions given by formulas (34)-(37). The effective interactions were derived from the free $\mathrm{np}, \mathrm{pp}$, and nn interactions. These effective interactions then served as input for the large-basis shell-model calculations of the $A=3$ system, e.g., ${ }^{3} \mathrm{H}$ and ${ }^{3} \mathrm{He}$, and of the $A=6$ system, e.g., ${ }^{6} \mathrm{Li},{ }^{6} \mathrm{He},{ }^{6} \mathrm{Be}$. In the present calculations we employed the CD-Bonn [4 potential, which includes isospin symmetry breaking terms. In addition, the Coulomb potential was added to the protonproton interaction. To get energy convergence, one needs $N_{\max }>30$ for the three-nucleon problem. However, for the purpose of the present work it is enough to perform the shellmodel calculations in a sufficiently large, but still easily accessible model space. We chose $N_{\max }=8$, which corresponds to $8 \hbar \Omega$ excitations above the unperturbed ground state for the $A=3$ system and $6 \hbar \Omega$ excitations for the $A=6$ system. For the harmonic-oscillator frequency, we picked $\hbar \Omega=19 \mathrm{MeV}$ for the $A=3$ system and $\hbar \Omega=17.2 \mathrm{MeV}$ for the $A=6$ system. These choices follow from the phenomenological formula $\hbar \Omega=45 A^{-\frac{1}{3}}-25 A^{-\frac{2}{3}}$ $\mathrm{MeV}$.

The wave-functions obtained in the no-core shell-model approach satisfy all the requirements necessary for realistic nuclear wave functions, i.e., the excitation of the center-of-mass of the nucleus is under control and they correspond to exact eigenvalues with good quantum numbers $J, \Pi$, (and $T$ in the case of the isospin invariant effective interaction). The results of our shell-model calculations are presented in Table I. These results demonstrate that, in general, the suggested definitions for the nucleon mass and the NN interactions are successful. The deviations between the exact (with charge-symmetry breaking interactions) and the approximate (with isospin invariant interactions) calculations are at the level of a fraction of a percent. The best correspondence between the results for the charge-dependent and charge-independent Hamiltonians is obtained for ${ }^{3} \mathrm{H}$ and ${ }^{3} \mathrm{He}$ and for all six states of ${ }^{6} \mathrm{Li}$. Moreover, we performed calculations for the $\mathrm{T}=1$ states in ${ }^{6} \mathrm{Li}$, using the isoscalar potential for $\mathrm{T}=0$. Analogous calculations for the $\mathrm{T}=0$ states of the same nucleus were also performed utilizing the isoscalar potential for $T=1$. In all cases the absolute values of differences between charge-dependent and charge-independent results are some three times larger in comparison with calculations using the correct value of the isospin for the state under investigation. As can be seen from the results in Table I, the biggest difference between energies is obtained for ${ }^{6} \mathrm{Be}$, where it equals $1-2 \%$ of the "exact" value, and, to some extent, in ${ }^{6} \mathrm{He}$, where it does not exceed $1 \%$. 


\section{SUMMARY AND CONCLUSIONS}

As is well-known, charge symmetry in nuclei is broken at the very least due to the difference between the proton and neutron masses, the charge-dependence of the strong interaction and the Coulomb interaction among the protons. These effects are not very large for light nuclei in comparison with the binding energy of the entire nucleus, but there are cases, in which they are significant and play a very important role, such as for nuclei near drip-lines, which are loosely bound. However, the description of nuclei using a fully charge-dependent Hamiltonian is complicated, so the construction of a Hamiltonian, as close as possible to charge-independent one, would be useful.

The present article is devoted to the consideration of this problem. It is shown that charge-dependence of the realistic Hamiltonian for light nuclei can be taken into account by replacing the realistic one by an equivalent charge-independent Hamiltonian. The parameters of such a Hamiltonian (i.e., the nucleon mass and the NN potential) are nucleus and state dependent. Our results can be formulated as simple recommendations for the construction of such an isoscalar Hamiltonian:

1. Take the nucleon mass equal to

$$
m=\frac{1}{A}\left(N m_{n}+Z m_{p}\right)
$$

2. Take the realistic NN potential in states with two-nucleon isospin $t=0$ as they are defined from the neutron-proton data analyze.

3. Take the isoscalar NN potential in states with $t=1$ as a linear combination, Eq. (32), of the neutron-neutron $\left(m_{t}=1\right)$, neutron-proton $\left(m_{t}=0\right)$, and proton-proton (with Coulomb potential included) $\left(m_{t}=-1\right)$ realistic potentials with coefficients $c_{t m_{t}}$, given in Eqs. (34)-(37).

The eigenfunctions of such a Hamiltonian have the isospin $T$ of the nucleus as good a quantum number, but are different for the individual members of the isospin multiplet due to the dependence of the isoscalar Hamiltonians on $M_{T}$.

In general, the results obtained are self-consistent and do not conflict with the simple requirements mentioned in the Introduction. Moreover, the isoscalar part of the Coulomb interaction has a nicer form than the earlier result suggested in $[16]$, Eq. (2.21), because the isoscalar Coulomb interaction of the nucleons in the present work equals zero in two-nucleon states with isospin $t=0$. Consequently, the normalization of this interaction to the number of proton pairs occurs naturally.

Our results can be considered to be quite good in all cases considered, because, due to our normalization conditions, Eq. (33), we really have only two free parameters for the construction of the isoscalar interaction. The approximation given here is a good starting

point for a perturbational account of nuclear charge-dependent effects not included in the isoscalar Hamiltonian. 


\section{ACKNOWLEDGMENTS}

This work was supported by NSF grants No. PHY96-05192 and INT98-06614. P.N. also acknowledges partial support from a grant of the Grant Agency of the Czech Republic 202/96/1562. G.P.K. acknowledges the CIES for a Fulbright Research Fellowship while at the University of Arizona and partial support from a grant No 391 of the Lithuanian State Science and Studies Foundation. G.P.K. and R.K.K. would like to thank Bruce R. Barrett and the University of Arizona for their hospitality.

\section{APPENDIX A}

The orthogonality of the transformation Eq. (14) follows directly from the observation that an arbitrary Jacobi tree can be written as a system of two subtrees, Fig. 3. The first (left) subtree with first-degree vertices $\mathbf{r}_{1} m_{1}, \mathbf{r}_{2} m_{2}, \ldots, \mathbf{r}_{q} m_{q}$ defines the Jacobi variables $\xi_{2}, \xi_{3}, \ldots, \xi_{q}$, the second (right) subtree contains $\mathbf{r}_{q+1} m_{q+1}, \mathbf{r}_{q+2} m_{q+2}, \ldots, \mathbf{r}_{q+t} m_{q+t}$ and $\xi_{q+1}, \xi_{q+2}, \ldots, \xi_{q+t-1}$, correspondingly. Obviously, it is possible to introduce in the first and second cases the additional Jacobi variables proportional to the center-of-mass coordinates of the subtrees, defined as

$$
\xi_{-1}=\frac{1}{\sqrt{m_{1 \ldots q}}} \sum_{i=1}^{q} \sqrt{m_{i}} \mathbf{x}_{i} ; \quad \text { and } \quad \xi_{-q}=\frac{1}{\sqrt{m_{q+1 \ldots q+t}}} \sum_{i=q+1}^{q+t} \sqrt{m_{i}} \mathbf{x}_{i} .
$$

The indices of these coordinates are negative, according to the rule that the arbitrary centerof-mass Jacobi variable situated near an intrinsic Jacobi variable $\xi_{\alpha}$ is labelled as $\xi_{1-\alpha}\left(\xi_{1}\right.$ and $\xi_{0}, \xi_{2}$ and $\xi_{-1}, \xi_{q+1}$ and $\left.\xi_{-q}\right)$. Suppose, the transformations from $\mathbf{r}_{1} m_{1}, \mathbf{r}_{2} m_{2}, \ldots, \mathbf{r}_{q} m_{q}$ to $\xi_{-1}, \xi_{2}, \xi_{3}, \ldots, \xi_{q}$ and from $\mathbf{r}_{q+1} m_{q+1}, \mathbf{r}_{q+2} m_{q+2}, \ldots, \mathbf{r}_{q+t} m_{q+t}$ to $\xi_{-q}, \xi_{q+1}, \xi_{q+2}, \ldots, \xi_{q+t-1}$ are orthogonal. Then the complete transformation is also orthogonal, because the transformation matrix from $\xi_{-1}, \xi_{-q}$ to $\xi_{0}, \xi_{1}$ is orthogonal:

$$
\left(\begin{array}{l}
\xi_{0} \\
\xi_{1}
\end{array}\right)=\left(\begin{array}{cc}
\sqrt{\frac{m_{1 \ldots q}}{M}} & \sqrt{\frac{m_{q+1 \ldots q+t}}{M}} \\
\sqrt{\frac{m_{q+1 \ldots q+t}}{M}} & -\sqrt{\frac{m_{1 \ldots q}}{M}}
\end{array}\right)\left(\begin{array}{l}
\xi_{-1} \\
\xi_{-q}
\end{array}\right) .
$$

Following this process to the top of the tree, we can arrive at orthogonal two-dimensional transformations. Therefore, the complete transformation $(q+t) \times(q+t)$ is orthogonal.

\section{APPENDIX B}

The independence of the matrix element, Eq. (16), $\left\langle\mathcal{E} J \Pi M_{T}\left|\nabla_{\eta_{\alpha}}^{2}\right| \mathcal{E} J \Pi M_{T}\right\rangle$, of $\alpha$, the intrinsic Jacobi variable number $(\alpha=1,2, \ldots, A-1)$, can be shown as follows. By definition, this element equals the product of sums over spin and isospin variables and of integrals over intrinsic Jacobi variables

$$
\begin{aligned}
& \sum_{\sigma_{1}, \sigma_{2} \ldots \sigma_{A}} \sum_{\tau_{1}, \tau_{2} \ldots \tau_{A}} \int d \eta_{1} \int d \eta_{2} \ldots \int d \eta_{A-1} \Psi_{\mathcal{E} J \Pi M_{T}}^{*}\left(\eta_{1} \eta_{2} \ldots \eta_{A-1} \sigma_{1,} \sigma_{2} \ldots \sigma_{A} \tau_{1}, \tau_{2} \ldots \tau_{A}\right) \\
& \times \nabla_{\eta_{\alpha}}^{2} \Psi_{\mathcal{E} J \Pi M_{T}}\left(\eta_{1} \eta_{2} \ldots \eta_{A-1} \sigma_{1}, \sigma_{2} \ldots \sigma_{A} \tau_{1}, \tau_{2} \ldots \tau_{A}\right)
\end{aligned}
$$


where $\Psi_{\mathcal{E} J \Pi M_{T}}(\ldots)$ is an intrinsic wave function of an atomic nucleus. This wave function is antisymmetric with respect to all nucleons. However, this property in Jacobi variables is not expandable in a simple way, because permutations of one-nucleon variables $\mathbf{r}_{i}$ generate orthogonal transformations of Jacobi variables. We can simplify by multiplying the given expression by the integral

$$
\int d \eta_{0} \Theta^{*}\left(\eta_{0}\right) \Theta\left(\eta_{0}\right) \equiv 1
$$

where $\Theta\left(\eta_{0}\right)$ is an arbitrary, normalized wave function of the center-of-mass. The new wave functions

$$
\Theta\left(\eta_{0}\right) \Psi_{\mathcal{E} J \Pi M_{T}}\left(\eta_{1} \eta_{2} \ldots \eta_{A-1} \sigma_{1}, \sigma_{2} \ldots \sigma_{A} \tau_{1}, \tau_{2} \ldots \tau_{A}\right)
$$

can be rewritten as usual antisymmetric functions of one-particle variables

$$
\Phi_{\mathcal{E} J \Pi M_{T}}\left(\mathbf{r}_{1} \mathbf{r}_{2} \ldots \mathbf{r}_{A} \sigma_{1}, \sigma_{2} \ldots \sigma_{A} \tau_{1}, \tau_{2} \ldots \tau_{A}\right) .
$$

Due to the orthogonality of the matrix a , Eq. (8), the integrals can be transformed into one-particle variables:

$$
\int d \eta_{0} \int d \eta_{1} \int d \eta_{2} \ldots \int d \eta_{A-1}=\int d \mathbf{r}_{1} \int d \mathbf{r}_{2} \ldots \int d \mathbf{r}_{A}
$$

The operator can be written in the form

$$
\nabla_{\eta_{\alpha}}^{2}=\sum_{i, j=1}^{A} a_{\alpha, i} a_{\alpha, j}\left(\nabla_{i} \cdot \nabla_{j}\right)=\sum_{i=1}^{A} a_{\alpha, i} a_{\alpha, i} \nabla_{i}^{2}+\sum_{i, j=1(i \neq j)}^{A} a_{\alpha, i} a_{\alpha, j}\left(\nabla_{i} \cdot \nabla_{j}\right) .
$$

The mean value of this operator is simply

$$
\begin{aligned}
\left\langle\nabla_{\eta_{\alpha}}^{2}\right\rangle \equiv & \sum_{\sigma_{1}, \sigma_{2} \ldots \sigma_{A}} \sum_{\tau_{1}, \tau_{2} \ldots \tau_{A}} \int d \mathbf{r}_{1} \int d \mathbf{r}_{2} \ldots \int d \mathbf{r}_{A} \Phi_{\mathcal{E} J \Pi M_{T}}^{*}\left(\mathbf{r}_{1} \mathbf{r}_{2} \ldots \mathbf{r}_{A} \sigma_{1}, \sigma_{2} \ldots \sigma_{A} \tau_{1}, \tau_{2} \ldots \tau_{A}\right) \\
& \times \nabla_{\eta_{\alpha}}^{2} \Phi_{\mathcal{E} J \Pi M_{T}}\left(\mathbf{r}_{1} \mathbf{r}_{2} \ldots \mathbf{r}_{A} \sigma_{1}, \sigma_{2} \ldots \sigma_{A} \tau_{1}, \tau_{2} \ldots \tau_{A}\right) \\
= & \sum_{i=1}^{A} a_{\alpha, i} a_{\alpha, i}\left\langle\nabla_{i}^{2}\right\rangle+\sum_{i, j=1(i \neq j)}^{A} a_{\alpha, i} a_{\alpha, j}\left\langle\left(\nabla_{i} \cdot \nabla_{j}\right)\right\rangle .
\end{aligned}
$$

Due to the antisymmetry of the wave functions, the matrix elements $\left\langle\nabla_{i}^{2}\right\rangle$ and $\left\langle\left(\nabla_{i} \cdot \nabla_{j}\right)\right\rangle$ are the same for all possible values of $i$ and $j$. Thus,

$$
\begin{aligned}
\left\langle\nabla_{\eta_{\alpha}}^{2}\right\rangle & =\left\langle\nabla_{A}^{2}\right\rangle \sum_{i=1}^{A} a_{\alpha, i} a_{\alpha, i}+\left\langle\left(\nabla_{A-1} \cdot \nabla_{A}\right)\right\rangle \sum_{i, j=1(i \neq j)}^{A} a_{\alpha, i} a_{\alpha, j} \\
& =\left\langle\nabla_{A}^{2}\right\rangle+\left\langle\left(\nabla_{A-1} \cdot \nabla_{A}\right)\right\rangle\left[\sum_{i=1}^{A} a_{\alpha, i} \sum_{j=1}^{A} a_{\alpha, j}-\sum_{i=1}^{A} a_{\alpha, i} a_{\alpha, i}\right] \\
& =\left\langle\nabla_{A}^{2}\right\rangle-\left\langle\left(\nabla_{A-1} \cdot \nabla_{A}\right)\right\rangle,
\end{aligned}
$$

because all sums are independent of $\alpha$, see Eqs. (9) and (10). Therefore, $\left\langle\nabla_{\eta_{\alpha}}^{2}\right\rangle$ is independent of $\alpha(\alpha=1,2, \ldots, A-1)$. 


\section{APPENDIX C}

The relation between potentials, defined in Eq. (32), holds also for the Reduced Hamiltonians $(\mathrm{RH})$ of the different two-nucleon states

$$
h^{0, j \pi t}=\sum_{m_{t}} c_{t m_{t}} h^{j \pi t m_{t}}
$$

and their matrix elements

$$
h_{\varepsilon, \epsilon^{\prime}}^{0, j \pi t}=\sum_{m_{t}} c_{t m_{t}} h_{\varepsilon, \epsilon^{\prime}}^{j \pi t m_{t}}
$$

because the partial-wave expressions for the kinetic energy operators are the same in all cases. For this reason, the difference of a diagonal matrix element of the Hamiltonian $H$ and an eigenvalue of the Hamiltonian $H^{0}$ for an arbitrary eigenstate of $H^{0}$ equals

$$
\begin{aligned}
\left\langle H-H^{0}\right\rangle_{\mathcal{E}, \mathcal{E}}^{J \Pi T M_{T}}= & \frac{A(A-1)}{2} \\
& \times \sum_{\varepsilon, \varepsilon^{\prime}, j \pi t m_{t}} h_{\varepsilon, \varepsilon^{\prime}}^{j \pi t m_{t}}\left\{\begin{array}{c}
Q_{\varepsilon^{\prime}, j \pi t m_{t} ; \varepsilon, j \pi t m_{t}}\left(\mathcal{E} J \Pi T M_{T}\right) \\
-\delta_{\varepsilon^{\prime}, \varepsilon} c_{t m_{t}} Q_{\varepsilon, j \pi t}(\mathcal{E} J \Pi T)
\end{array}\right\} .
\end{aligned}
$$

There are nondiagonal contributions to the charge-dependent density matrix, because the eigenfunctions of the three RH operators $h^{j \pi t m_{t}}\left(m_{t}=0, \pm 1\right)$ are different and are not equal to the eigenfunctions of the isoscalar $\mathrm{RH} h^{0, j \pi t}$. The matrix of the isoscalar $\mathrm{RH}$ in this case is diagonal, i.e.,

$$
h_{\varepsilon, \varepsilon^{\prime}}^{0, j \pi t}=\sum_{m_{t}} c_{t m_{t}} h_{\varepsilon, \varepsilon^{\prime}}^{j \pi t m_{t}}=\varepsilon^{j \pi t} \delta_{\varepsilon, \varepsilon^{\prime}}
$$

The left-hand side of Eq. (C1) is the sum of traces of products of two symmetrical matrices, the first of these is the matrix of the $\mathrm{RH}$ and the second is the reduced density matrix. As is seen from (C1), this sum contains only two kinds of terms - one is equal to the product of diagonal elements of both matrices, and the other is equal to the product of nondiagonal elements. The last subsum is independent of the choice of the values of variational coefficients $c_{t m_{t}}$. In any case, its contribution is negligible in comparison with the first subsum, which contains diagonal elements. This result is caused by the following reasons: The nondiagonal elements of the charge-dependent $\mathrm{RH}$ matrices are negligible in every two nucleon state, because charge-independence and charge-symmetry breaking forces in light atomic nuclei are significantly weaker than original charge-independent strong interaction between nucleons. The nondiagonal entries of charge-dependent density matrix are also small in absolute value, because eigenfunctions of the charge-dependent $\mathrm{RH}$ are close to eigenfunctions of charge-independent $\mathrm{RH}$ for the same reason as given previously.

The most important diagonal part of the sum in Eq. (C1) is

$$
\sum_{\varepsilon, j \pi t m_{t}} h_{\varepsilon, \varepsilon}^{j \pi t m_{t}}\left\{Q_{\varepsilon, j \pi t m_{t}}\left(\mathcal{E} J \Pi T M_{T}\right)-c_{t m_{t}} Q_{\varepsilon, j \pi t}(\mathcal{E} J \Pi T)\right\}
$$


The problem is to choose the coefficients $c_{t m_{t}}$ in such a way that this sum will have its minimal value. Let us first recall some definitions. The distributions of probabilities, given in Eqs. (23) and (30), differ. In the last expression they are comparable, because corresponding density matrices are created by the same wave-function. The relationship between these probabilities, following directly from the definition of the density matrix, is (see [1] and [13]):

$$
\sum_{m_{t}} Q_{\varepsilon, j \pi t m_{t}}\left(\mathcal{E} J \Pi T M_{T}\right)=Q_{\varepsilon, j \pi t}(\mathcal{E} J \Pi T)
$$

This equality means that the quantity on the right-hand side is a marginal probability (see, for example, [14). In the case $t=0$, all entries of $(\mathrm{C} 2)$ vanish, when $c_{00}=1$. This value is consistent with the definition of the isoscalar interaction, Eqs. (32), (33), because, as mentioned earlier, in this case we have no other choice, excluding the best one. In the case when $t=1$, the situation is not so simple, because only the sum of the three multipliers of $h_{\varepsilon, \varepsilon}^{j \pi t m_{t}}$, corresponding to the three possible values of the isospin projection $m_{t}$, vanishes, due to relations (33) and (C3). In general, for any value of $t$ the relation:

$$
\sum_{m_{t}}\left\{Q_{\varepsilon, j \pi t m_{t}}\left(\mathcal{E} J \Pi T M_{T}\right)-c_{t m_{t}} Q_{\varepsilon, j \pi t}(\mathcal{E} J \Pi T)\right\}=0
$$

is satisfied.

This relation is an identity, and, hence, is not sufficient to define the coefficients, but gives us more choices for determining the minimal value of the sum given in Eq. (C2). The best choice occurs when the partial sum of the coefficients over all possible two-nucleon channels is equal to zero, namely,

$$
\sum_{\varepsilon, j \pi}\left\{Q_{\varepsilon, j \pi t m_{t}}\left(\mathcal{E} J \Pi T M_{T}\right)-c_{t m_{t}} Q_{\varepsilon, j \pi t}(\mathcal{E} J \Pi T)\right\}=0
$$

This equation can also be expressed in the form

$$
Q_{t m_{t}}\left(T M_{T}\right)=c_{t m_{t}} Q_{t}(T) .
$$

The marginal probabilities are defined by

$$
\sum_{\varepsilon, j \pi} Q_{\varepsilon, j \pi t m_{t}}\left(\mathcal{E} J \Pi T M_{T}\right)=Q_{t m_{t}}\left(T M_{T}\right)
$$

and

$$
\sum_{m_{t}} Q_{t m_{t}}\left(T M_{T}\right)=Q_{t}(T)
$$

and can be extracted from the expressions for the simple multiparticle operators with known eigenvalues, such as the mass number $A$, the charge $Z$ and the total isospin $T$, (see [11], [13). Therefore, 


$$
\begin{aligned}
Q_{00}\left(T M_{T}\right) & \equiv Q_{0}(T)=\frac{A(A+2)-4 T(T+1)}{4 A(A-1)}, \\
Q_{1}(T) & =\frac{3 A(A-2)+4 T(T+1)}{4 A(A-1)}, \\
Q_{1-1}\left(T M_{T}\right) & =\frac{\left(A-2 M_{T}\right)\left(A-2 M_{T}-2\right)}{4 A(A-1)}, \\
Q_{10}\left(T M_{T}\right) & =\frac{A(A-2)+4 T(T+1)-8 M_{T}^{2}}{4 A(A-1)}, \\
Q_{1+1}\left(T M_{T}\right) & =\frac{\left(A+2 M_{T}\right)\left(A+2 M_{T}-2\right)}{4 A(A-1)} .
\end{aligned}
$$

Using Eq. (C4), we obtain

$$
c_{t m_{t}}=Q_{t m_{t}}\left(T M_{T}\right) / Q_{t}(T) .
$$

Applying this expression, we obtain the values of the coefficients given in Eqs. (34)-(37). 


\section{REFERENCES}

[1] J. Carlson and R. Schiavilla, Rev. Mod. Phys. 70, 743 (1998).

[2] V. G. J. Stoks, R. A. M. Klomp, C. P. F. Terheggen, and J. J. de Swart, Phys. Rev. C 49, 2950 (1994).

[3] R. B. Wiringa, V. G. J. Stoks, and R. Schiavilla, Phys. Rev. C 51, 38 (1995).

[4] R. Machleidt, F. Sammarruca, and Y. Song, Phys. Rev. C 53, 1483 (1996).

[5] R. V. Reid, Jr., Ann. Phys. (N. Y.) 50, 411 (1968).

[6] J. L. Friar and B. F. Gibson, Phys. Rev. C 17, 1456 (1978).

[7] J. L. Friar, B. F. Gibson, and G. L. Payne, Phys. Rev. C 36, 1140 (1987).

[8] Ju. F. Smirnov and K. V. Shitikova, Sov. J. Part. Nucl. 8, 847 (1977).

[9] G. P. Kamuntavičius, Few-Body Systems 1, 91 (1986).

[10] A. Bohr and B. Mottelson, Nuclear structure (W. A. Benjamin, Inc. New York, Amsterdam, 1969), Vol. 1, Ch. 2.

[11] G. P. Kamuntavičius, Sov. J. Nucl. Phys. 34, 368 (1981).

[12] G. P. Kamuntavičius, Sov. J. Part. Nucl. 20, 261 (1989).

[13] A. Bernotas, A. Bičkutè, and G. P. Kamuntavičius, Soviet Physics Collection (Lietuvos Fizikos Rinkinys), 23, 22 (1983).

[14] W. Feller, An introduction to probability theory and its applications (John Willey \& Sons, 1970) Vol. 1, Ch. 9.

[15] P. Navrátil and B. R. Barrett, Phys. Rev. C 54, 2986 (1996); Phys. Rev. C 57, 3119 (1998).

[16] B. S. Pudliner, V. R. Pandparipande, J. Carlson, Steven C. Pieper, and R. B. Wiringa, Phys. Rev. C 56, 1720 (1997). 
TABLE I. Comparison of energies, in $\mathrm{MeV}$, obtained in the no-core shell-model calculations with the full charge-dependent effective interactions, $\mathrm{E}_{P-N}$, and the corresponding isospin-invariant effective interactions, $\mathrm{E}_{I S O}$ (as defined in the text), for $\mathrm{A}=3$ and $\mathrm{A}=6$ nuclei. The effective interactions were derived from the CD-Bonn potential [4]. Harmonicoscillator energies of $\hbar \Omega=19 \mathrm{MeV}$ and $\hbar \Omega=17.2 \mathrm{MeV}$ were employed for the $\mathrm{A}=3$ and $\mathrm{A}=6$ systems, respectively. For $A=3$ a complete $8 \hbar \Omega$ model-space was utilized, while for $A=6$ a complete $6 \hbar \Omega$ model-space was used.

\begin{tabular}{llll}
\hline \hline${ }^{A} \mathrm{Z}\left(J^{\pi} T\right)$ & $\mathrm{E}_{P-N}$ & $\mathrm{E}_{I S O}$ & {$\left[E_{P-N}-E_{I S O}\right] / E_{P-N}$} \\
\hline${ }^{3} \mathrm{H}\left(\frac{1}{2}^{+} \frac{1}{2}\right)$ & -8.441 & -8.421 & $-2.44 \times 10^{-3}$ \\
${ }^{3} \mathrm{He}\left(\frac{1}{2}^{+} \frac{1}{2}\right)$ & -7.668 & -7.643 & $-3.27 \times 10^{-3}$ \\
${ }^{6} \mathrm{He}\left(0^{+} 1\right)$ & -25.665 & -25.830 & $+6.42 \times 10^{-3}$ \\
${ }^{6} \mathrm{He}\left(2^{+} 1\right)$ & -22.944 & -23.085 & $+6.13 \times 10^{-3}$ \\
${ }^{6} \mathrm{Li}\left(1^{+} 0\right)$ & -28.257 & -28.180 & $-2.73 \times 10^{-3}$ \\
${ }^{6} \mathrm{Li}\left(3^{+} 0\right)$ & -25.605 & -25.525 & $-3.12 \times 10^{-3}$ \\
${ }^{6} \mathrm{Li}\left(0^{+} 1\right)$ & -24.815 & -24.666 & $-6.02 \times 10^{-3}$ \\
${ }^{6} \mathrm{Li}\left(2^{+} 0\right)$ & -23.146 & -23.071 & $-3.25 \times 10^{-3}$ \\
${ }^{6} \mathrm{Li}\left(2^{+} 1\right)$ & -21.934 & -21.924 & $-4.47 \times 10^{-4}$ \\
${ }^{6} \mathrm{Li}\left(1^{+} 0\right)$ & -20.433 & -20.362 & $-3.46 \times 10^{-3}$ \\
${ }^{6} \mathrm{Be}\left(0^{+} 1\right)$ & -22.829 & -22.545 & $-1.25 \times 10^{-2}$ \\
${ }^{6} \mathrm{Be}\left(2^{+} 1\right)$ & -20.227 & -19.851 & $-1.86 \times 10^{-2}$ \\
\hline \hline
\end{tabular}




\section{A. Figure captions}

FIG. 1. An example of a Jacobi tree.

FIG. 2. The Jacobi tree for a nucleus composed of $\mathrm{Z}$ protons and $\mathrm{N}$ neutrons.

FIG. 3. An example of a Jacobi tree with subtrees. 


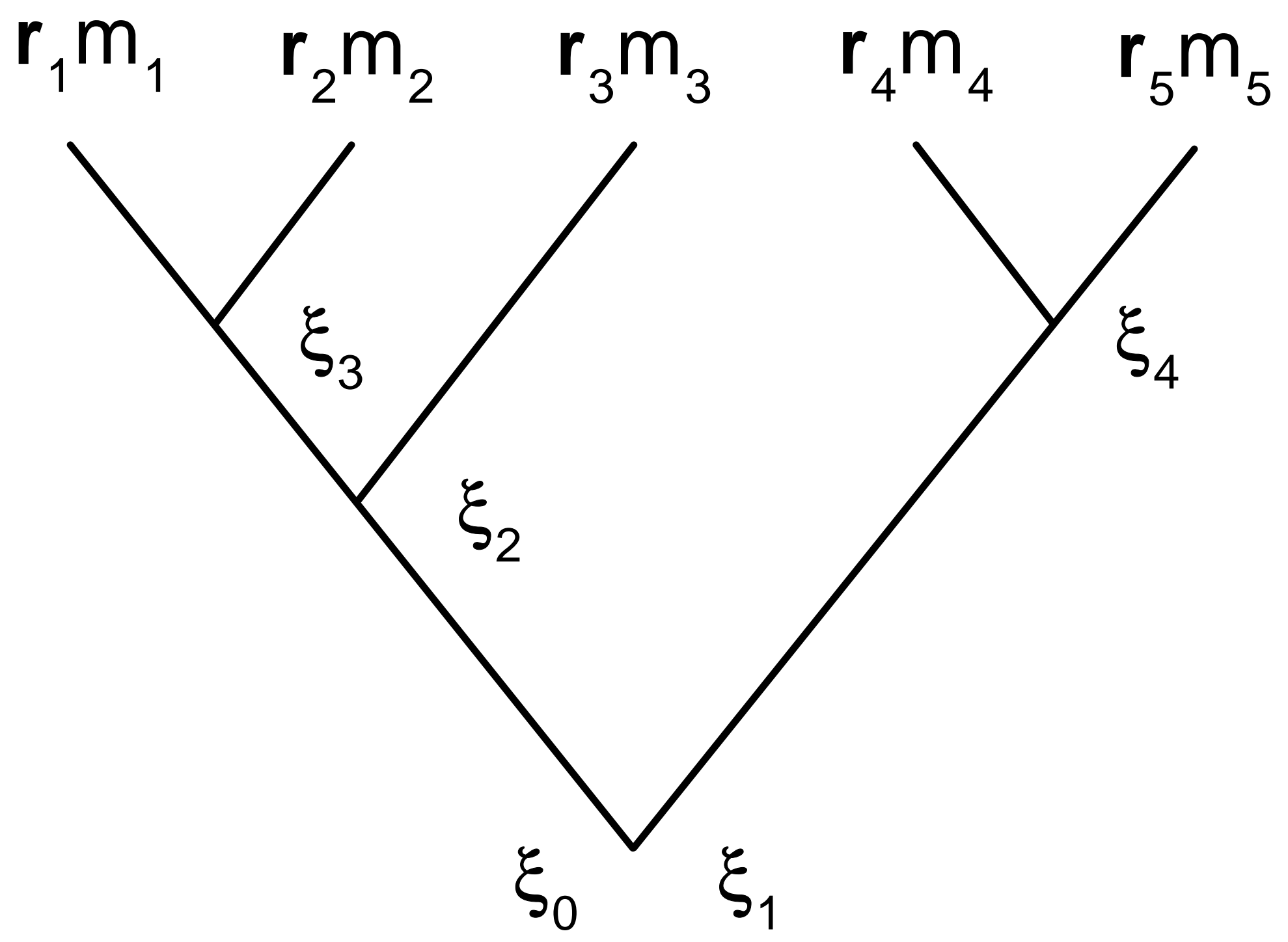

FIG. 1. The Jacobi tree sample.

Isoscalar Hamiltonians for light atomic nuclei G.P.Kamuntavicius, B.R.Barrett, P.Navratil, G.Sapragonaite, and R.K.Kalinauskas 


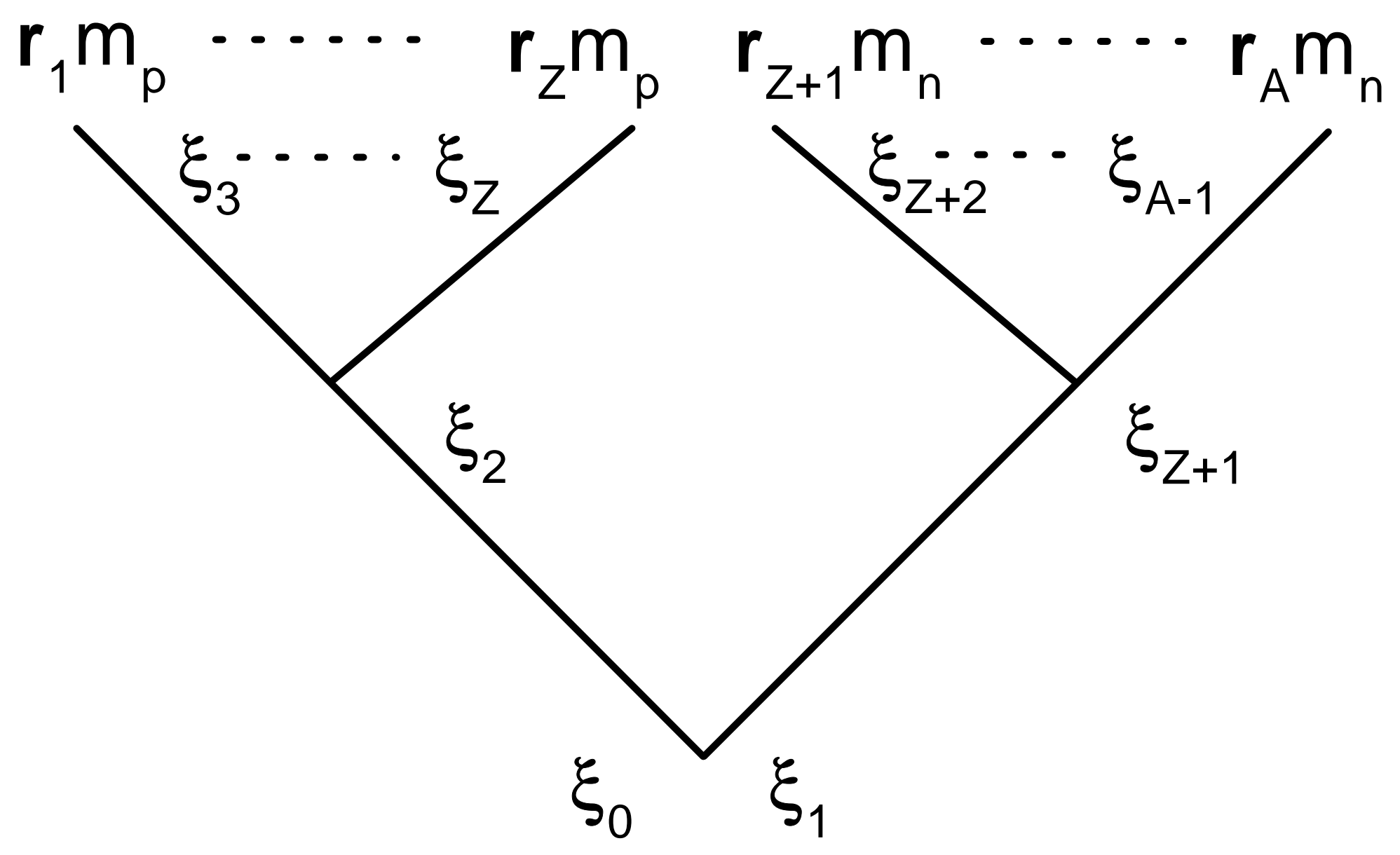

FIG. 2. The Jacobi tree for nucleus composed of $Z$ protons and $\mathrm{N}$ neutrons.

Isoscalar Hamiltonians for light atomic nuclei

G.P.Kamuntavicius, B.R.Barrett, P.Navratil,

G.Sapragonaite, and R.K.Kalinauskas 


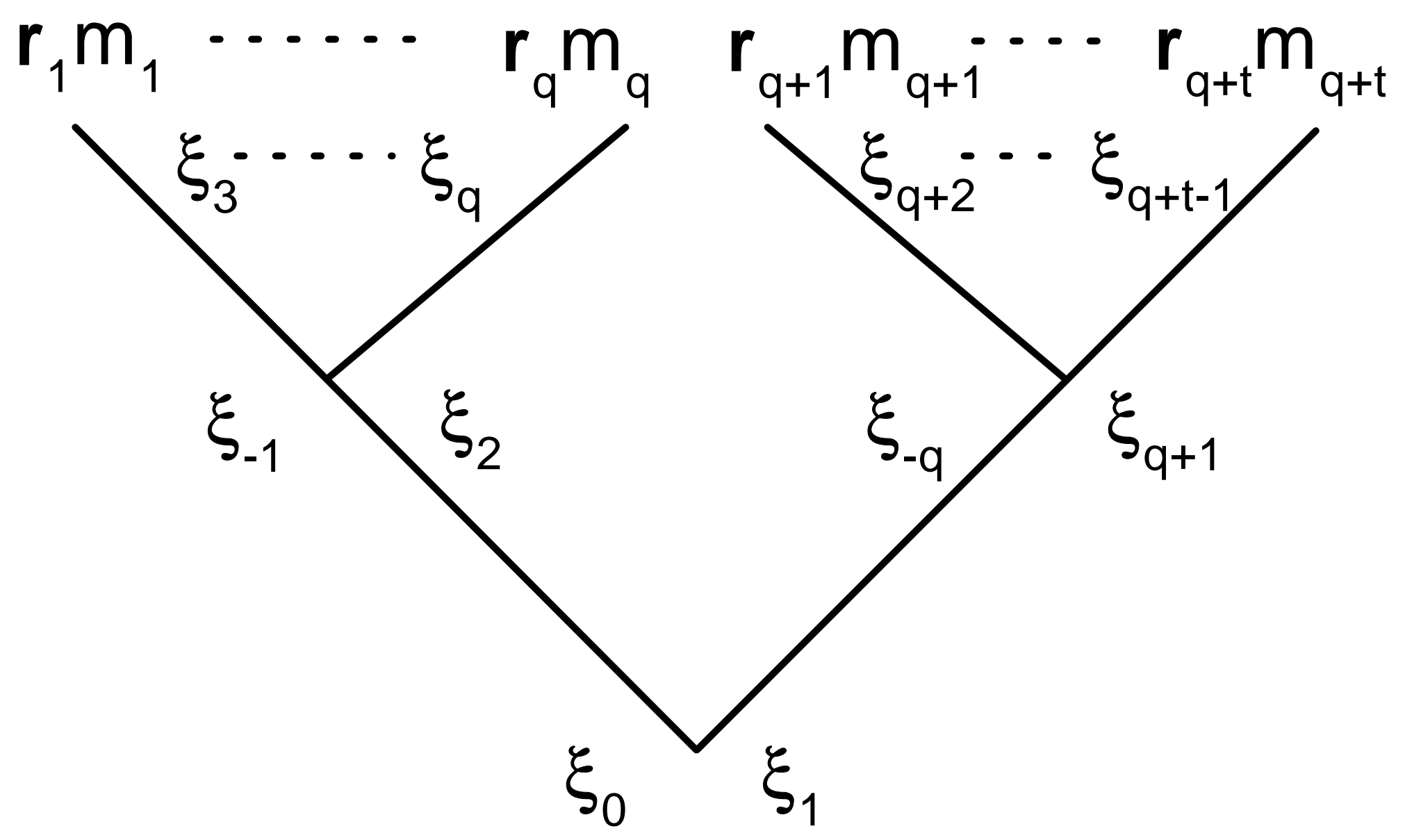

FIG. 3. The subtrees of Jacobi tree.

Isoscalar Hamiltonians for light atomic nuclei G.P.Kamuntavicius, B.R.Barrett, P.Navratil, G.Sapragonaite, and R.K.Kalinauskas 